\title{
Evidence For Two Separate But Interlaced Components of the Chromospheric Magnetic Field
}

\author{
K. P. Reardon ${ }^{1}$ \\ Osservatorio Astrofisico di Arcetri, 50125 Firenze, Italy; kreardon@arcetri.astro.it \\ Y.-M. Wang \\ Space Science Division, Naval Research Laboratory, Washington, DC 20375-5352, USA; \\ yi.wang@nrl.navy.mil \\ K. Muglach ${ }^{2}$ \\ NASA Goddard Space Flight Center, Greenbelt, MD 20771, USA; karin.muglach@nasa.gov \\ and \\ H. P. Warren \\ Space Science Division, Naval Research Laboratory, Washington, DC 20375-5352, USA; \\ hwarren@nrl.navy.mil
}

\begin{abstract}
Chromospheric fibrils are generally thought to trace out low-lying, mainly horizontal magnetic fields that fan out from flux concentrations in the photosphere. A high-resolution ( $\sim 0^{\prime \prime} 1$ per pixel) image, taken in the core of the Ca II $854.2 \mathrm{~nm}$ line and covering an unusually large area, shows the dark fibrils within an active region remnant as fine, looplike features that are aligned parallel to each other and have lengths comparable to a supergranular diameter. Comparison with simultaneous line-of-sight magnetograms confirms that the fibrils are centered above intranetwork areas (supergranular cell interiors), with one end rooted just inside the neighboring plage or strong unipolar network but the other endpoint less clearly defined. Focusing on a particular arcade-like structure lying entirely on one side of a filament channel (large-scale polarity inversion), we find that the total amount of positive-polarity flux underlying this "fibril arcade" is
\end{abstract}

\footnotetext{
${ }^{1}$ Also at Astrophysics Research Centre, Queen's University, Belfast, BT7 1NN, Northern Ireland, UK.

${ }^{2}$ Also at ARTEP, Inc., Ellicott City, MD 21042, USA.
} 
$\sim 50$ times greater than the total amount of negative-polarity flux. Thus, if the fibrils represent closed loops, they must consist of very weak fields (in terms of total magnetic flux), which are interpenetrated by a more vertical field that contains most of the flux. This surprising result suggests that the fibrils in unipolar regions connect the network to the nearby intranetwork flux, while the bulk of the network flux links to remote regions of the opposite polarity, forming a second, higher canopy above the fibril canopy. The chromospheric field near the edge of the network thus has an interlaced structure resembling that in sunspot penumbrae.

Subject headings: Sun: chromosphere — Sun: corona — Sun: faculae, plages — Sun: magnetic topology — Sun: surface magnetism

\section{Introduction}

The solar chromosphere is permeated by a variety of fine, elongated structures, whose physical nature and relationship to each other and to the overlying corona are not well understood. These features include the forest of $\mathrm{H} \alpha$ spicules that protrude above the limb, the dark fibrils and mottles (on-disk spicules?) that cover much of the disk, and the long filaments that lie along polarity inversion lines of the photospheric magnetic field (see, e.g., Beckers 1968; Veeder \& Zirin 1970; Foukal 1971a, 1971b; Suematsu et al. 1995; Martin 1998; De Pontieu et al. 2004; Rutten 2007). In addition, the term "dynamic fibril" has been used to describe the relatively short $(\sim 1-4 \mathrm{Mm})$ jets, with velocities of order $20 \mathrm{~km} \mathrm{~s}^{-1}$ and lifetimes of 3-6 minutes, which are associated with active region plage and the transition region "moss" seen in Fe IX 17.1 nm (De Pontieu et al. 2007; Hansteen et al. 2006). Hereinafter, we restrict the definition of fibrils to include only the long, horizontal structures that form canopies over supergranular cell centers and have individual lifetimes $\gtrsim 10$ minutes. Shorter, less highly inclined, and more dynamical features are considered to be "spicules," whether they occur on the disk or above the limb.

Fibrils are best observed with very narrow bandpass in the cores of lines such as $\mathrm{H} \alpha$ and Ca II 854.2 nm (Cauzzi et al. 2008; Reardon et al. 2009); in the wings of these lines, they appear as truncated stalks, suggesting the presence of upflows and downflows. Similar structures, extending to greater heights, are seen in the extreme ultraviolet (EUV) in He II $30.4 \mathrm{~nm}$ and Fe IX $17.1 \mathrm{~nm}$, where they again appear in absorption. Fibrils are generally assumed to consist of relatively dense material aligned along nearly horizontal magnetic field lines that fan out from photospheric flux concentrations, with the (not necessarily visible) ends of the flux tubes being rooted in opposite polarities. They exhibit a large-scale 
organization in and around active regions and their unipolar remnants, but tend to be shorter and more randomly oriented in the quiet Sun.

A puzzling question concerns the behavior of fibrils inside predominantly unipolar magnetic regions: where are the endpoints of the other polarity located? To address this question, we compare the fibril structure in a decaying active region, imaged with unprecedented spatial resolution in Ca II $854.2 \mathrm{~nm}$, with the line-of-sight component of the underlying photospheric field. This comparison leads to some surprising inferences about the nature of the fibril fields.

\section{Observations}

The Interferometric Bidimensional Spectrometer (IBIS) is a ground-based imaging spectrometer that utilizes a pair of Fabry-Perot interferometers to record very narrow-band filtergrams in the spectral range 580-860 nm (Cavallini 2006; Reardon \& Cavallini 2008; Righini et al. 2010). The instrument, installed on the Dunn Solar Telescope at the National Solar Observatory (NSO/Sacramento Peak), has a 95"-diameter field of view and a resolution of $\sim 0^{\prime \prime} 1$ per pixel. Between 15:03 and 15:43 UT on 2010 August 3, IBIS acquired images of an active region (NOAA 11092) located just northward of disk center, in a variety of spectral lines (including $\mathrm{Ca}$ II $854.2 \mathrm{~nm}$ and $\mathrm{H} \alpha$ ) and at multiple positions within each line profile. For each wavelength position, the $3 \times 3$ subfields spanning the active region were reconstructed using the Kiepenheuer-Institut Speckle Interferometry Package (Wöger et al. 2008) and assembled into a single $240^{\prime \prime} \times 240^{\prime \prime}$ mosaic. Our analysis focuses on the composite image constructed from observations in the line core of Ca II $854.2 \mathrm{~nm}$, which shows fibril structures at a height of $\sim 1.2 \mathrm{Mm}$ above the photosphere with unusual clarity over such a large field of view. The overwhelming dominance of the fibrils in the Ca II $854.2 \mathrm{~nm}$ line core is mainly due to the use of a very narrow bandpass $(4.5 \mathrm{pm})$, which isolates the chromospheric contribution effectively from the much stronger photospheric radiation (Reardon et al. 2009).

For comparison purposes, we also employ simultaneous observations made with the Helioseismic and Magnetic Imager (HMI) and the Atmospheric Imaging Assembly (AIA) on the Solar Dynamics Observatory $(S D O)$, as described below. The spatial resolution of the $S D O$ full-disk images is substantially lower than that of the IBIS observations, being 0..5 per pixel for the HMI magnetograms and 0.'6 for the AIA EUV filtergrams. 


\section{Relation to the Photospheric Magnetic Field}

The two panels of Figure 1 display the Ca II $854.2 \mathrm{~nm}$ mosaic and the underlying distribution of photospheric magnetic flux. The latter was obtained by averaging 66 line-ofsight magnetograms recorded between 14:51 and 15:45 UT with SDO/HMI, after correcting for the effect of solar rotation. It is apparent that the dark fibrils tend to be centered above areas of relatively low magnetic flux density (neutral gray in Figure 1(b)), including in particular the supergranular cell interiors. This well-known canopy-like behavior is consistent with the assumption that the fibrils trace out low-lying horizontal field lines that fan outward from the edges of both large and small photospheric flux concentrations. The fibrils in the active region remnant have a remarkably "well-combed" appearance, forming individual clumps that stream in the same direction, away from the areas of strong plage toward the quieter background. Thus, the fibrils are organized not only on the supergranular scale, but also on a much larger ("circumfacular") scale surrounding the core of the active region itself (see, e.g., Bumba \& Howard 1965; Harvey 2006; Wang et al. 2011).

One immediate impression obtained from the high-resolution Ca II $854.2 \mathrm{~nm}$ filtergram is that the individual fibrils have a looplike appearance. Surrounding the sunspot, long, curved structures (sometimes called "threads") clearly link plage of opposite polarity. Elsewhere, however, the fibrils seem to have only one endpoint located in plage or strong unipolar network (where they are relatively bright), while the other endpoint is difficult to identify. Especially perplexing is the behavior of the many fibrils that span unipolar cells (examples of such cells are circled in Figure 1). Do these fibrils pass over the opposite side of the cell, becoming invisible in Ca II $854.2 \mathrm{~nm}$ and in $\mathrm{H} \alpha$ and ending up in some remote area of the opposite polarity (see Foukal 1971a, 1971b)? Or do they connect to weak intranetwork flux just before reaching the opposite boundary of the cell? A closely related question concerns the total amount of magnetic flux contained in the fibrils.

The rectangular box in Figure 1 encloses a striking feature which we hereafter refer to as a "fibril arcade." This system of looplike fibrils lies along the eastern side of a filament channel that separates the positive-polarity sector of the active region remnant from the negative-polarity sector to the west. The easternmost ends of the fibrils are rooted just inside the strong positive-polarity plage; instead of connecting to the negative-polarity plage on the other side of the filament channel, however, the fibrils bend sharply toward the southwest until they run parallel to the channel. Their western ends, like those of the adjacent fibrils in the filament channel, appear to be located inside the weak intranetwork region.

Assuming that the fibril arcade indeed consists of closed loops, we may obtain an upper limit for the total magnetic flux in the fibrils by summing up all of the minority-polarity (negative) flux underlying the arcade. Figure 2 shows a close-up of the fibril arcade and 
the corresponding line-of-sight photospheric field, where in this case we have taken a 4.5minute average of HMI magnetograms recorded at approximately the same time that the fibril arcade was imaged. We find that only $2 \%$ of the photospheric flux underlying the arcade (as defined by the dashed lines) has negative polarity; the overwhelming majority of the flux within the dashed polygon has positive polarity and is concentrated near the edge of the plage along the eastern and southern boundary of the arcade. It follows that at most $4 \%$ of the total unsigned flux underlying the arcade is associated with the fibril fields, while at least $96 \%$ of the flux (including most of the positive-polarity flux near the edge of the plage) must penetrate through the arcade and close elsewhere. Note that this general result is insensitive to the exact positioning of the dashed lines in Figure 2, requiring only that the southeastern boundary of the arcade lie within the positive-polarity plage and that its northwestern boundary be located in the adjacent non-plage area. Clearly, if the arcade were taken to extend farther into the plage region, the percentage of interpenetrating flux would be even greater.

Figure 3 shows the distribution of coronal emission in the vicinity of the fibril arcade, as recorded in Fe XII $19.3 \mathrm{~nm}$ with SDO/AIA. Patches of strong Fe XII emission (formed at a temperature of $\sim 1.3 \mathrm{MK}$ ) overlie the Ca II $854.2 \mathrm{~nm}$ arcade, as is apparent from the superposed images in Figure 3(d). Most of these brightenings occur above flux concentrations along the edge of the plage region, and we interpret them as marking the locations of coronal loops that penetrate through the fibril arcade, analogous to the "moss" observed in Fe IX $17.1 \mathrm{~nm}$ (Berger et al. 1999).

From Figures 1 through 3, it is evident that the Ca II $854.2 \mathrm{~nm}$ fibrils tend to be relatively bright where they are anchored in plage and strong network. This partial "fillingin" of the line core (which remains in absorption) indicates the presence of enhanced heating where the magnetic field is strong (see Cauzzi et al. 2009), perhaps due to the generation of magnetoacoustic waves and shocks (Hansteen et al. 2006; De Pontieu et al. 2007) or to the effect of radiative heat transfer to the fibril material from the higher-temperature coronal loops.

To make it clear that the chromospheric field is extremely nonpotential, Figure 4 displays a current-free extrapolation of the photospheric field in the region surrounding the fibril arcade. Here, the solar surface is approximated as flat, periodic boundary conditions are applied along the sides of the Cartesian computational box, and Laplace's equation is solved using the Fourier method. Unlike the observed fibril structures, most of the calculated field lines originating along the edge of the positive-polarity plage connect directly to the negativepolarity plage on the other side of the filament channel. The nonpotential nature of the fibril fields is hardly surprising, given that they are confined to an atmospheric layer where $\beta$, the 
ratio of plasma to magnetic pressure, is of order unity (see, e.g., Judge 2006).

\section{Fibrils Inside Unipolar Regions}

We now consider the fibril structures that lie entirely within unipolar regions. Figure 5 shows a close-up of the large positive-polarity cell circled in the lower left quadrant of Figure 1. The clumps of Ca II $854.2 \mathrm{~nm}$ fibrils rooted along the northern and southern boundaries of this circular cell all stream southward (Figure 5(a)), away from the area where the positive-polarity plage has its greatest concentration (see Figure 5(b)). The cell interior is thus blanketed by fibrils that originate either from the network along the northern boundary of the cell or from the local intranetwork. The visual impression given by the Ca II $854.2 \mathrm{~nm}$ image is that most of these fibrils do not pass through or over the southern boundary of the cell, but remain confined to the cell interior; moreover, they appear to be continuous rather than sharply cut-off or open-ended structures. From the magnetogram in Figure 5(b), it is again evident that the total amount of positive-polarity flux underlying the fibrils greatly exceeds the total amount of negative-polarity flux. As in the preceding example, we deduce that the bulk of the network flux underlying the fibrils penetrates through them into the corona, and that the fibrils themselves contain a relatively small amount of flux whose southern endpoints are located in the intranetwork region. Figure 5(c) shows the IBIS observations of the same region recorded in the core of the $\mathrm{H} \alpha$ line. Like their Ca II $854.2 \mathrm{~nm}$ counterparts, the $\mathrm{H} \alpha$ fibrils emanating from the northern edge of the cell seem to be confined to the cell interior. In contrast, as seen from Figure 5(d), the long Fe IX $17.1 \mathrm{~nm}$ loops that originate from the same area extend well beyond the southern perimeter of the cell.

In the upper left (northeast) corner of the images in Figure 5, a circular "hole" may be seen inside the dense positive-polarity plage. This small supergranular cell is likewise covered by dark Ca II $854.2 \mathrm{~nm}$ and $\mathrm{H} \alpha$ fibrils which appear to link the plage to weak negative-polarity flux inside the cell.

Based on comparisons between $\mathrm{H} \alpha$ filtergrams and relatively low-resolution magnetograms, Foukal (1971a, 1971b) argued that H $\alpha$ fibrils anchored in unipolar network cannot connect across supergranular cells, and must therefore be open-ended absorbing features that are visible only along a limited portion of the entrained flux tubes, like spicules and mottles. Our analysis, employing much higher-quality data, suggests instead that Ca II $854.2 \mathrm{~nm}$ and $\mathrm{H} \alpha$ fibrils are associated with the small fraction of the network flux that connects to the nearby intranetwork region. Such flux tubes would be characterized by very large inclinations to the vertical. Less inclined flux tubes representing the bulk of the unipolar network would 
penetrate into the corona and develop a chromospheric-coronal transition region, closing in a remote area of the opposite polarity.

In images taken in the wings of Ca II $854.2 \mathrm{~nm}$ and $\mathrm{H} \alpha$, the fibrils appear as truncated stalks rooted along the plage and network boundaries, with the stalks tending to be longer and darker in the blue wing than in the red. This suggests the presence of upflows, downflows, and/or thermal broadening due to enhanced heating at the strong-field footpoints of the fibrils, with the direction of flow being predominantly away from the network toward the intranetwork regions. As in the case of the "dynamic fibrils" described and modeled by De Pontieu et al. (2004, 2007) and Hansteen et al. (2006), the mass-loading of the horizontal fibrils might be driven by the leakage of p-mode wave energy into the chromosphere along highly inclined field lines.

\section{Summary and Discussion}

Our main conclusions may be stated as follows:

1. Fibrils observed in the line core of Ca II $854.2 \mathrm{~nm}$ (and of $\mathrm{H} \alpha$ ) have the appearance of fine looplike structures with characteristic lengths on the order of a supergranular diameter.

2. In the active region remnant studied here, the fibrils generally have one end rooted just inside strong unipolar network and the other end located in the neighboring intranetwork region.

3. The total magnetic flux in the fibrils is much less than the total underlying flux.

4. Thus most of the underlying flux must penetrate through the fibril canopy.

5. As it fans out into the corona, the interpenetrating network flux must form its own canopy above the low-lying fibril canopy. Most of the flux in this larger-scale canopy connects to remote areas of the opposite polarity.

The chromospheric field near the edge of unipolar network therefore consists of at least two distinct components: a horizontal fibril field that connects the network to the adjacent intranetwork region, and a more vertical field that contains most of the flux and penetrates through the fibrils into the corona. Spicules, mottles, and dynamic fibrils would be associated mainly with the less inclined component.

The proposed magnetic topology near the edge of the network bears a remarkable re-

semblance to the "interlocking comb" structure of sunspot penumbrae. There, the dark penumbral filaments are highly inclined toward the vertical and plunge back into the pho- 
tosphere, while the interspersed bright filaments are directed upward into the corona (see, e.g., Figure 4 in Weiss et al. 2004).

An implicit assumption in our analysis is that the (unsigned) flux density in the intranetwork, which depends on the spatial resolution of the magnetogram, is small compared to that in the network. In the case shown in Figure 2, the breakdown of this assumption would require the actual flux in the intranetwork to be $\sim 50$ times greater than in the 0.5 resolution HMI data. Based on an inspection of higher-resolution Hinode magnetograms, we consider this possibility to be unlikely.

Because of the highly nonpotential nature of the chromosphere, the existence or nonexistence of a magnetic canopy (see Schrijver \& Title 2003; Aiouaz \& Rast 2006; Jendersie \& Peter 2006) cannot be deduced directly from current-free extrapolations of the photospheric field. Observationally, it is clear that the fibrils do form canopies over the supergranular cell interiors, although these low-lying canopies contain only a small fraction of the network flux and are riddled with holes. Unfortunately, it may be difficult to measure the weak fibril fields directly using current spectropolarimetry methods; thus, the reasonable supposition that the fibrils trace out magnetic field lines has yet to be definitively tested.

We thank G. Cauzzi and N. R. Sheeley, Jr. for stimulating discussions, and the AIA and HMI science teams for providing the SDO data. IBIS was built by INAF/OAA with contributions from the University of Florence, University of Rome, MIUR, and MAE, and is operated with the support of NSO. This work was funded by NASA, NSF, and ONR. 


\section{REFERENCES}

Aiouaz, T., \& Rast, M. P. 2006, ApJ, 647, L183

Beckers, J. M. 1968, Sol. Phys., 3, 367

Berger, T. E., De Pontieu, B., Schrijver, C. J., \& Title, A. M. 1999, ApJ, 519, L97

Bumba, V., \& Howard, R. 1965, ApJ, 141, 1492

Cauzzi, G., et al. 2008, A\&A, 480, 515

Cauzzi, G., Reardon, K., Rutten, R. J., Tritschler, A., \& Uitenbroek, H. 2009, A\&A, 503, 577

Cavallini, F. 2006, Sol. Phys., 236, 415

De Pontieu, B., Erdélyi, R., \& James, S. P. 2004, Nature, 430, 536

De Pontieu, B., Hansteen, V. H., Rouppe van der Voort, L., van Noort, M., \& Carlsson, M. 2007, ApJ, 655, 624

Foukal, P. 1971a, Sol. Phys., 19, 59

Foukal, P. 1971b, Sol. Phys., 20, 298

Hansteen, V. H., De Pontieu, B., Rouppe van der Voort, L., van Noort, M., \& Carlsson, M. 2006, ApJ, 647, L73

Harvey, J. W. 2006, in Solar Polarization 4, ed. R. Casini \& B. W. Lites (ASP Conf. Ser. 358; San Francisco, CA: ASP), 419

Jendersie, S., \& Peter, H. 2006, A\&A, 460, 901

Judge, P. 2006, in Solar MHD Theory and Observations: A High Spatial Resolution Perspective, ed. J. Leibacher, R. F. Stein, \& H. Uitenbroek (ASP Conf. Ser. 354; San Francisco, CA: ASP), 259

Martin, S. F. 1998, Sol. Phys., 182, 107

Reardon, K. P., \& Cavallini, F. 2008, A\&A, 481, 897

Reardon, K. P., Uitenbroek, H., \& Cauzzi, G. 2009, A\&A, 500, 1239

Righini, A., Cavallini, F., \& Reardon, K. P. 2010, A\&A, 515, A85

Rutten, R. J. 2007, in The Physics of Chromospheric Plasmas, ed. P. Heinzel, I. Dorotovič, \& R. J. Rutten (ASP Conf. Ser. 368; San Francisco, CA: ASP), 27

Schrijver, C. J., \& Title, A. M. 2003, ApJ, 597, L165

Suematsu, Y., Wang, H., \& Zirin, H. 1995, ApJ, 450, 411

Wang, Y.-M., Robbrecht, E., \& Muglach, K. 2011, ApJ, 733, 20 
Weiss, N. O., Thomas, J. H., Brummell, N. H., \& Tobias, S. M. 2004, ApJ, 600, 1073

Wöger, F., von der Lühe, O., \& Reardon, K. 2008, A\&A, 488, 375

Veeder, G. J., \& Zirin, H. 1970, Sol. Phys., 12, 391

This preprint was prepared with the AAS LATEX macros v5.2. 


\section{(a) Ca II $854.2 \mathrm{~nm}$}

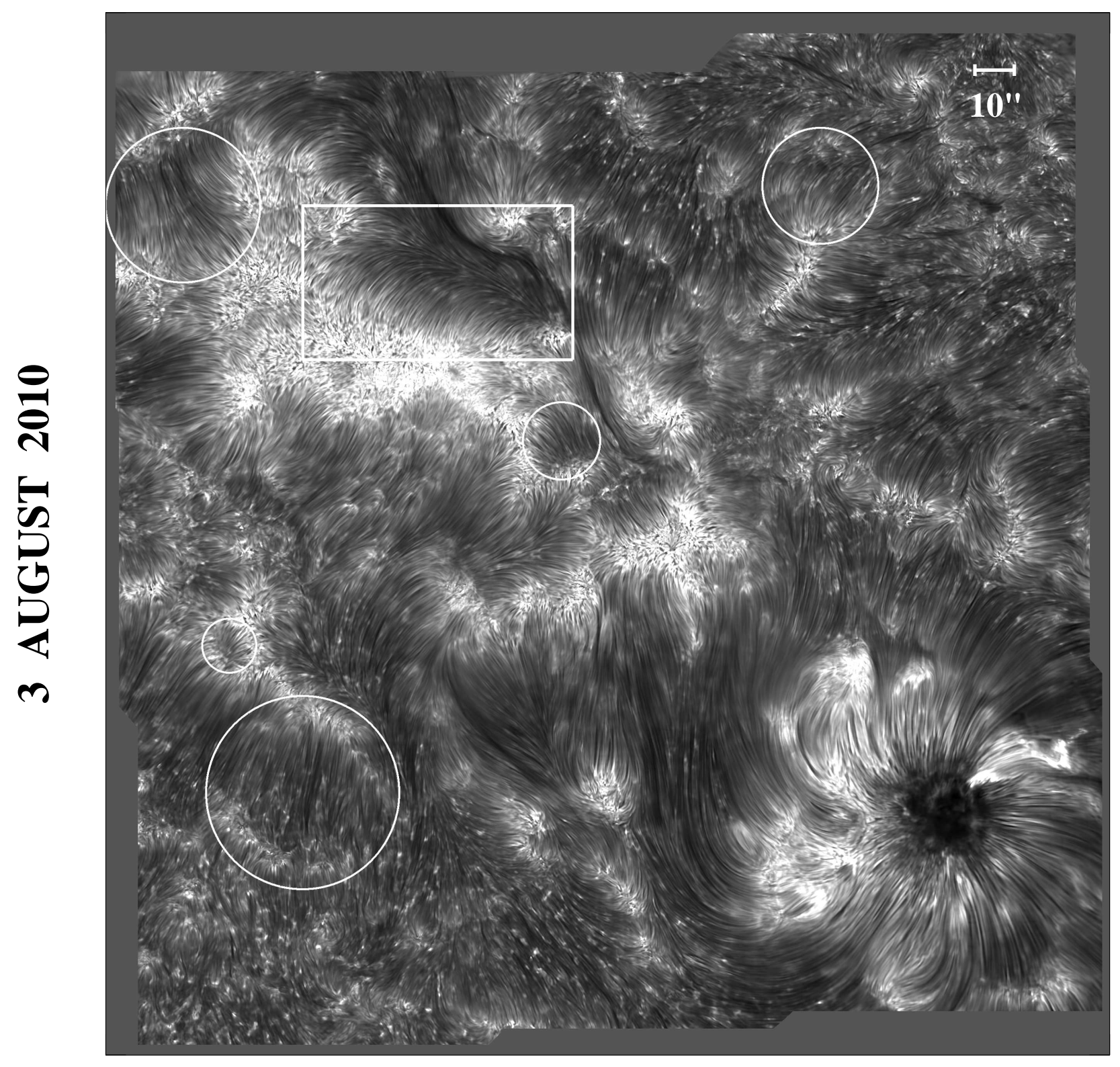




\section{(b) HMI $B_{\text {LOS }}(15 /-15$ G)}

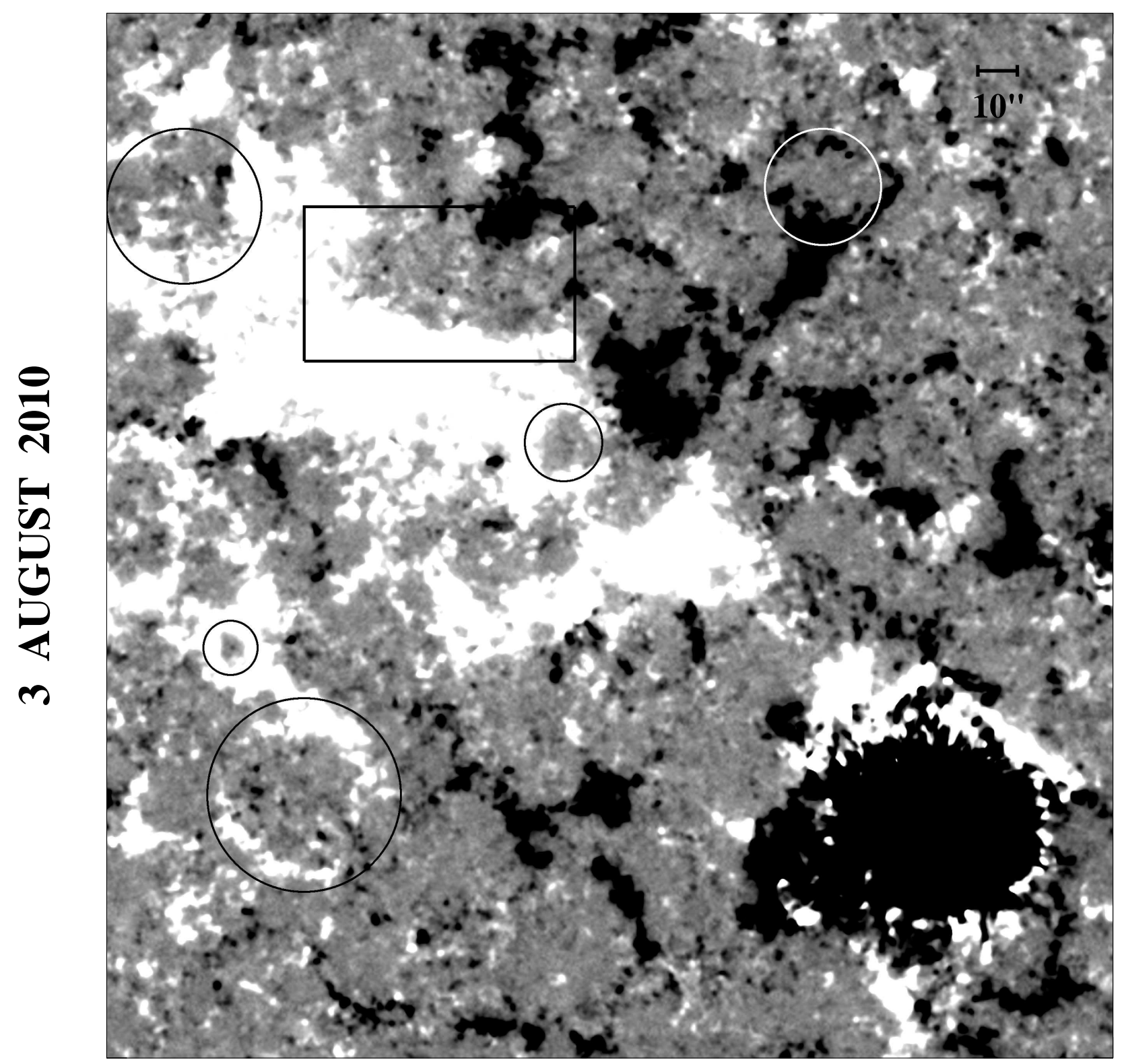

Fig. 1.- Active region (NOAA 11092) observed near central meridian on 2010 August 3. Field of view has dimensions $254^{\prime \prime} \times 264^{\prime \prime}$, with the sunspot in the southwest corner being located at latitude $12^{\circ} \mathrm{N}$. North is up; west is to the right. (a) Image taken in the line core of Ca II $854.2 \mathrm{~nm}$ at NSO/Sacramento Peak with IBIS. This mosaic was assembled from 9 subfields recorded between 15:03 and 15:43 UT; image scale is 0".098 per pixel and diffraction-limited resolution is 0.23 . (b) Underlying distribution of photospheric magnetic flux, obtained by averaging 66 line-of-sight magnetograms recorded with SDO/HMI between 14:51 and 15:45 UT. Gray scale ranges from $B_{\text {los }}<-15 \mathrm{G}$ (black) to $B_{\text {los }}>+15 \mathrm{G}$ (white); resolution is $\sim 1$ ". Boxed area contains the "fibril arcade" discussed in the text; examples of fibril-blanketed unipolar cells are circled. 


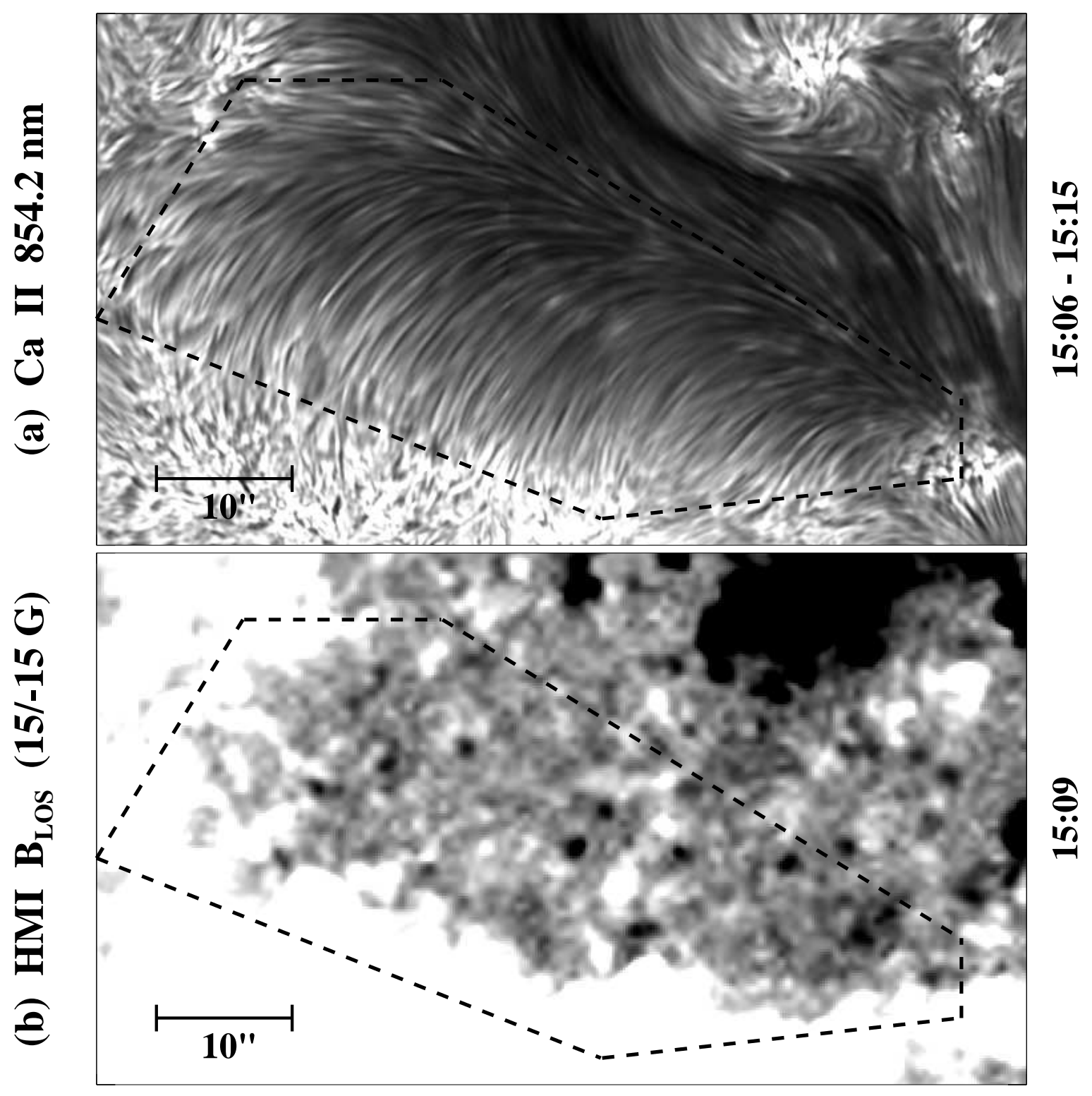

Fig. 2.- Close-up of the boxed area in Figure 1: (a) Ca II $854.2 \mathrm{~nm}$ fibril arcade; (b) underlying photospheric flux distribution. The line-of-sight magnetogram, saturated at $B_{\text {los }}= \pm 15 \mathrm{G}$, represents a 5-minute average of HMI measurements taken around the time when the arcade was imaged with IBIS ( 15:09 UT on 2010 August 3), rather than an hourlong average as in Figure 1(b). Inside the dashed boundary, the total amount of negativepolarity flux is only $2 \%$ of the total amount of positive-polarity flux. 

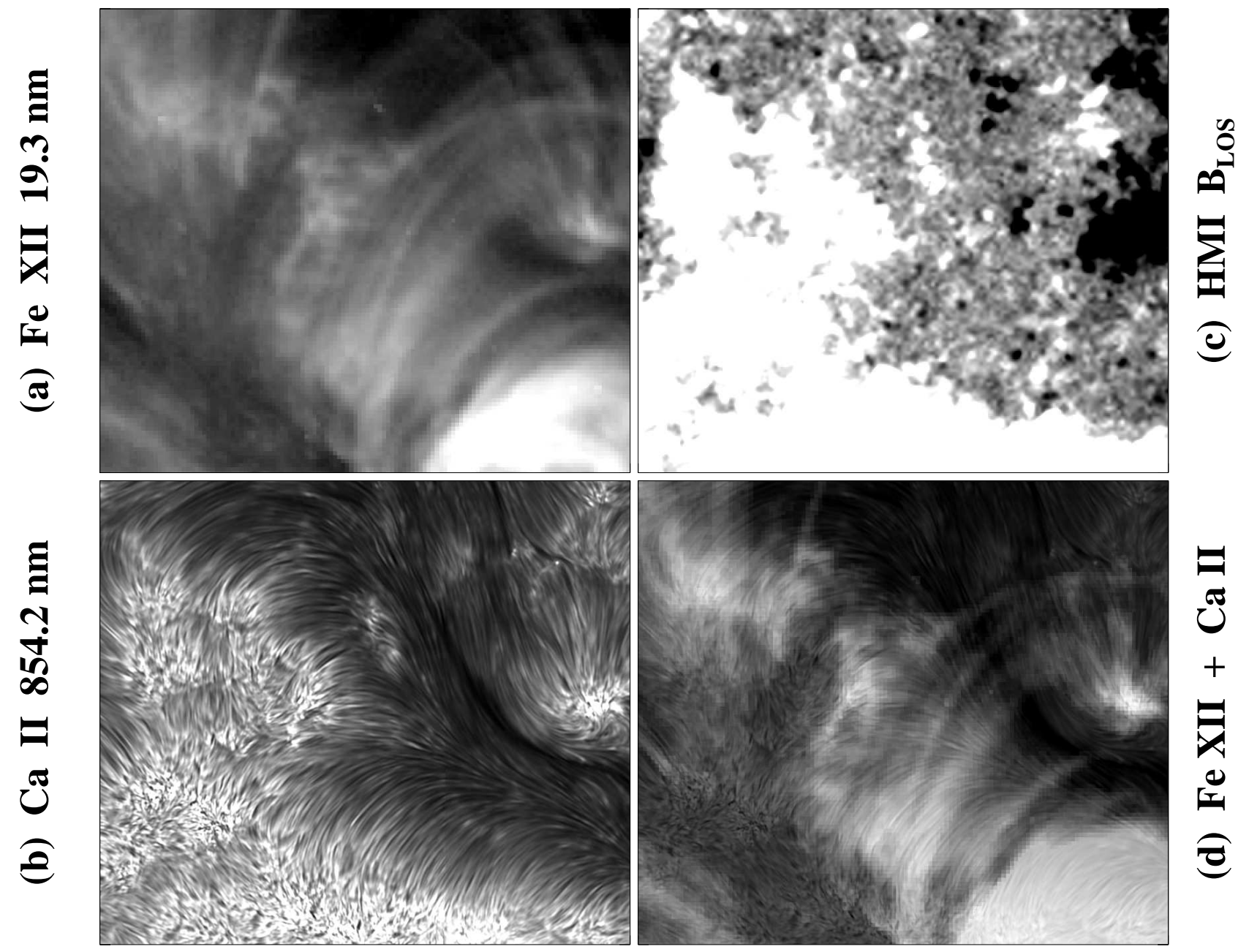

\begin{tabular}{l}
$\Xi$ \\
\multirow{0}{*}{} \\
+ \\
0 \\
0 \\
0 \\
0 \\
0
\end{tabular}

Fig. 3.- Coronal emission in the vicinity of the fibril arcade. (a) Fe XII $19.3 \mathrm{~nm}$ image recorded with SDO/AIA at 15:09 UT on 2010 August 3; plate scale is 0"6 per pixel. (b) Ca II $854.2 \mathrm{~nm}$ fibril arcade. (c) HMI line-of-sight magnetogram (5-minute average), saturated at $B_{\text {los }}= \pm 15$ G. (d) Superposition of the Fe XII $19.3 \mathrm{~nm}$ and Ca II $854.2 \mathrm{~nm}$ images. 


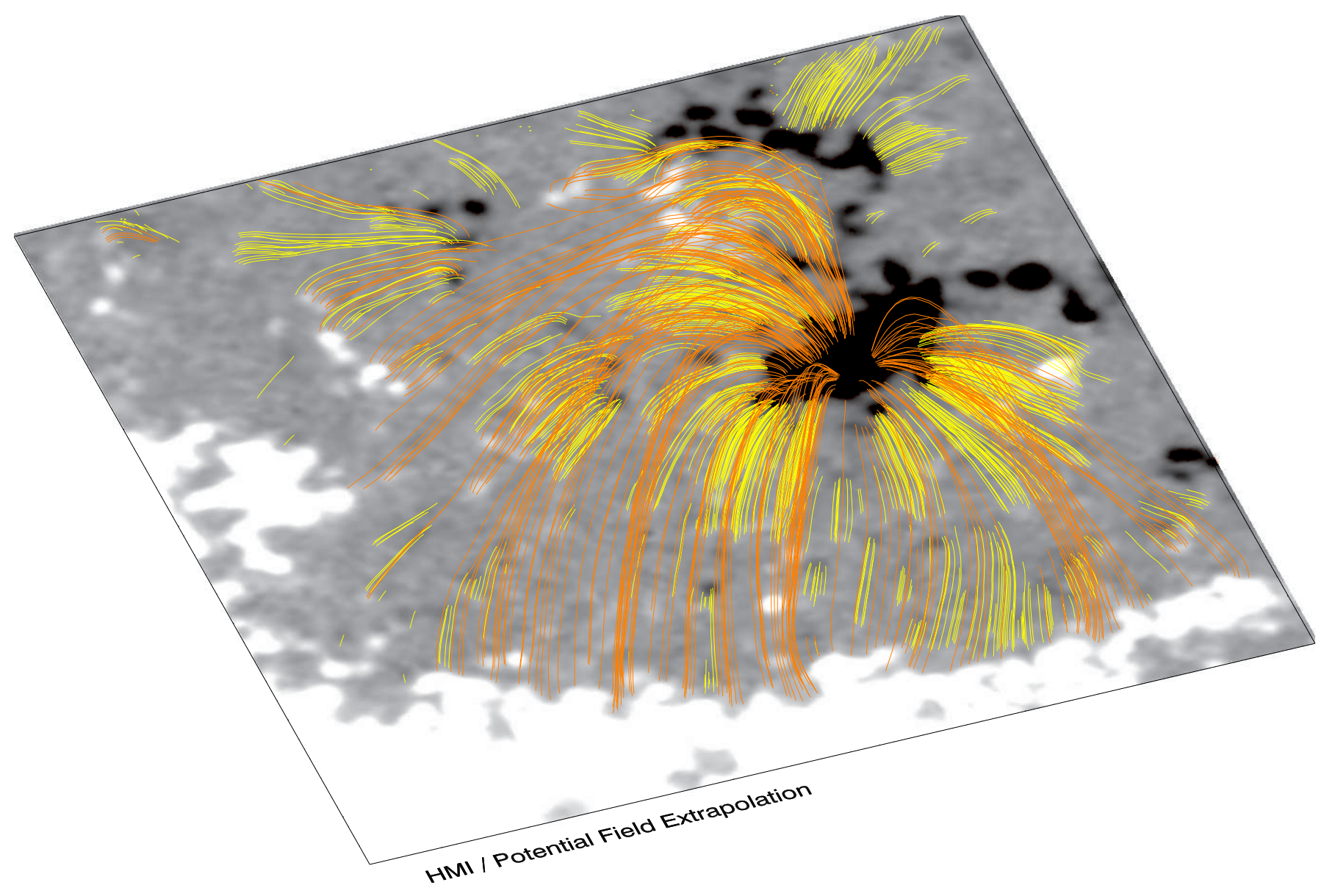

Fig. 4.- Potential field extrapolation of the HMI magnetogram, showing field lines (orange) connecting the edge of the positive-polarity plage to the negative-polarity plage. Short $\left(<15^{\prime \prime} \simeq 11 \mathrm{Mm}\right)$ field lines with at least one footpoint in the intranetwork are plotted in yellow. 


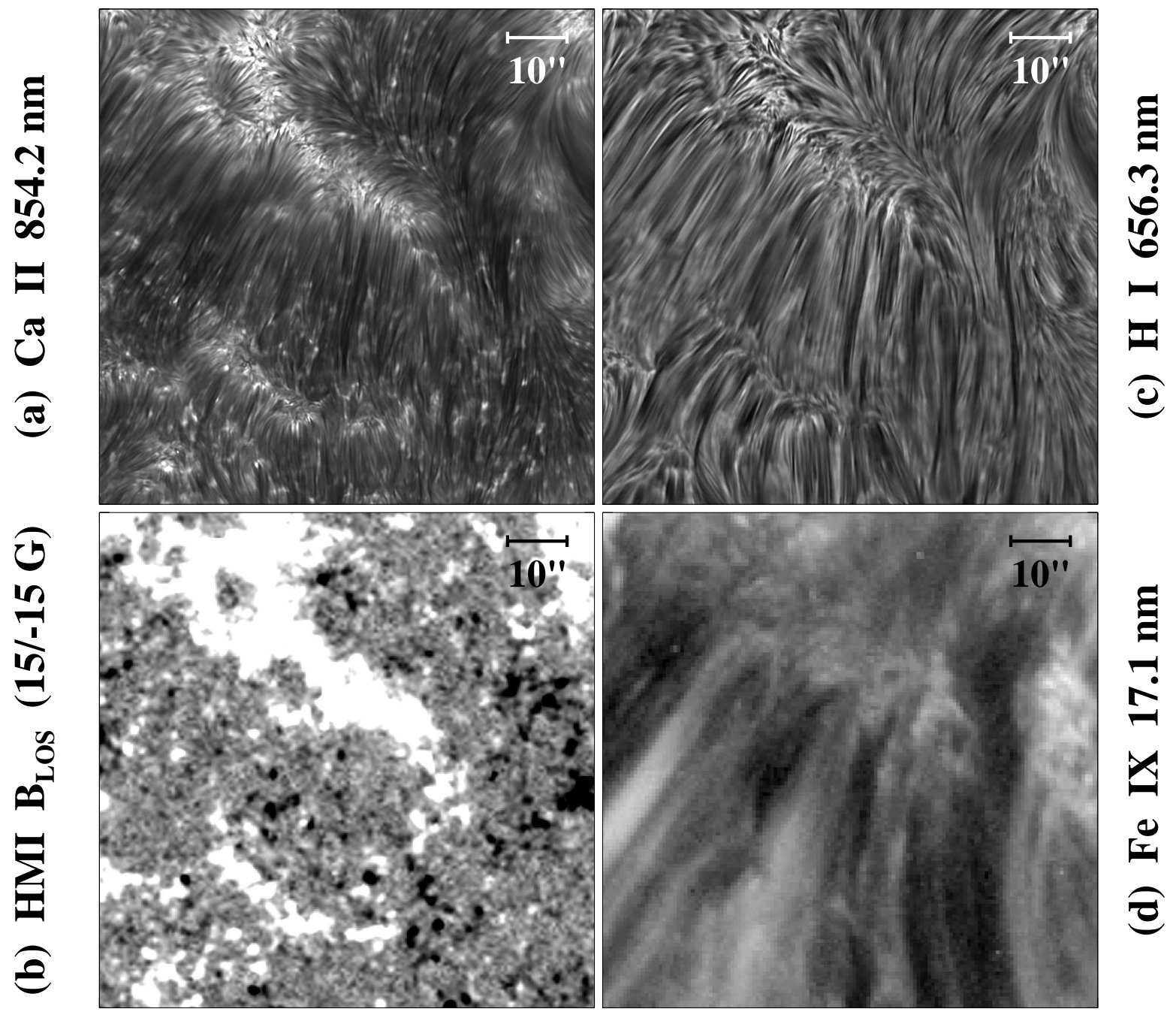

Fig. 5.- Close-up of unipolar supergranular cells located in the southeast quadrant of Figure 1. (a) Ca II 854.2 nm line core (IBIS, 15:11-15:27 UT). (b) Underlying line-of-sight photospheric field, saturated at $B_{\mathrm{los}}= \pm 15 \mathrm{G}$ (5-minute average of HMI magnetograms taken at 15:22 UT). (c) H $\alpha$ line core (IBIS, 15:11-15:27 UT). (d) Fe IX $17.1 \mathrm{~nm}$ image recorded with AIA at 15:21 UT. 